\title{
Theory of the Systematicism — Its Existence, Thinking and Essence
}

\author{
Yuan Hongjian \\ The School of Marxism, Wuhan University of Science and Technology, Wuhan, China \\ Email address: \\ hgdt2006@aliyun.com
}

\section{To cite this article:}

Yuan Hongjian. Theory of the Systematicism - lts Existence, Thinking and Essence. International Journal of Systems Science and Applied Mathematics. Vol. 3, No. 3, 2018, pp. 62-66. doi: 10.11648/j.ijssam.20180303.11

Received: September 17, 2018; Accepted: October 10, 2018; Published: November 12, 2018

\begin{abstract}
For the years, many scholars in the systematic theory field thought the "view of the system" same with the "view of the material", considering the material same with the system, the external world an objective existence independent from the will of the people. After more than half a century of exploration, people have gradually realized that the "view of the system" is different from the "view of the material", that the system is not the same thing with the material. The people have recognized that the view of the unified world is limited, that the world actually is broken, that the theory of the system existence is often associated with the concept of exchange. Specifically, the essence of the system existence is the exchange. The chain field thinking space and the seven-layer model deducted from the the concept of exchange are the logical result of the combination of the spiritual elements and the system. From the chain field thinking space and the seven-layer model, we can see the existence-thinking dual property phenomenon, which supplying the possibility to revolve the argue between the materialism and idealism. The $\mathrm{N}$ times of the circulation moved by the logical exchange in the system would produce the whole logical exchange chain in people's thinking, by which, people would get a kind of new taste of the ontology in the philosophy and a clear prospect of human's development through the study of the history related to the people's society and the world, both of which are multi-level, multi-fields, and multi-form.
\end{abstract}

Keywords: Systematism, Exchange, Chain Domain, Existence-Thinking Duality, Seven-Layer Model

\section{Introduction}

In the time of the last 1960s and 1970s, there were the so-called "old three theories", including cybernetics, information theory and the system theory, and the so called "new three theories" including dissipative structure theory, synergy theory and the catastrophe theory. Both the old and the new one, called as the system science or transverse science, ever disseminated rapidly in the global intellectual and thinking fields. In the late 1990s, the cybernetics and information theory among the "old three theories" have achieved considerable development and progress both in theory and practice. However, the system theory seemed encounter the bottlenecks, reflecting research difficulties and confusion. Although some progress and theory results has been achieved in the system theory, but there was no fundamental breakthrough, the system theory gradually involved to be the complex system, still waiting for their "Newton". [1]

\section{Confusion in the Research on the System Theory}

What are the main difficulties and confusion in the system theory study nowadays ? I think the first one should be the view of the system science.

The view of the system was regarded as the same thing with the view of the material in the early study on the system theory. People thought the system same as the material and there was an unified material world independent from the will of the human. [2] The world was considered systematic, with some belongs to this kind system and some to other kind. Influenced by the unified system theory, there has been no a widely accepted definition for the system although some progress achieved in terms of some concepts such as the layer, emerge, the self-organization, complete and the part. Without the widely accepted definition, the study of the system science should have a long way to go in the exploring the unknown 
cognition process and the natural rules. [Note 1]

With the situation mentioned above, many scholars in the world have transferred their research from the system theory to the complex system, considering the subject of the system science a very complicated cognition object, having the desire to find a path for the system theory. At the same time, some scholars argued that the complexity is a concept of attributes, like the one as "beauty" in the art, they thought the research on certain attributes may not contribute to resolving the main problem on the system theory. [3] Therefore, some scholars tried a different way, thinking that the main resistance in the system theory may lies in the understanding of the view of system. They argued that the world is not unified as thought before, but a broken one. It could be modified by people's study. Professor $\mathrm{Wu}$ Tong ${ }^{(1)}$ from Tsinghua University has published two papers in the Journal of Systems Science entitled as "The Broken View of System" and "The Entirety and the Broken", in which he wrote: "We can admit that there is an external world, but this world is the one for people's practice and understanding, not the abstract system." [4] Hawking $^{(2)}$ also pointed out in the Big Design published in 2011: "There is no picture- or theory-independent concept of reality". Hawking's meaning here refers to the modification as per people's cognition development. [5]

\section{Things Are of More Than Mutually Connected}

Relevant philosophy theories tell us that the world is a unified system in which all the things, phenomena, process and aspects interacting to each. [6] But, this kind explain is not enough. The theory of system exchange believes that things are not only connected or interacted but also exchanged by the way of exchange chain or the network. The elements in the exchange consist of not only the material but also the energy, information, time and space, even spirit or consciousness. The exchange chain or exchange network is also a system with evolution by the way such as production, development, getting merged or annexed, going spiraled up, decline, split or faded. The elements staying outside the evolution system we are searching on can be considered as non-systematic, then, the systems and non-systems would have the exchange interaction. This explain could be the basic framework for the system exchange theory.

Many philosophy theories argue that the world is of material, the material is of moving, the moving is the basic attribute of the material. The system exchange theory argues that the world is systematic, the system is inter-exchanged and the exchange is the basic attribute of the system. [7] In fact, in the process of our cognition and practice, many kinds of exchange phenomena whether from the nature or the conscious could be distinguished to be this kind system or that kind one. Conversely speaking, we must focus on the specific exchange phenomenon when we are talking about some system. So, by this meaning, the concept of system is closely related to the concept of exchange, neither of them can exist independently from each other. Therefore, the system exchange theory uses the concept of exchange as its core in defining the system and performing the discussion of system theory. Refers to the reason behind the exchange mentioned by some scholars, such as the self-organization or other-organization of the system, it is just another problem, just like the reason why Einstein's definition of the speed of the light is 300,000 kilometers per second. Here, what we focus on is just the system exchange theory, which could be regarded as a common law or phenomenon in the universe.

\section{System Exchange Theory}

The system exchange theory consists of one definition, three inferences, six basic exchange elements, one chain domain thinking space, and one seven-layer model, with the exchange or exchange chain as its core and the broken world as its point of view of the world.

Definition: The system generally has multiple exchange chains approaching to the external world, with at least one main exchange chain in a certain time and space. This main exchange chain dominates other exchange chains. Without the exchange chain or the main exchange chain, the system cannot be a system, or an independent and stable system. [7] [Note 2]

Inference 1 of the concept of system (original exchange): In the process of the system exchange interacting with the external world, there is always a trend of becoming bigger and stronger. Once there is an opportunity, the system would take the action such as decompose, merge, etc. [7] [Note 3]

Inference 2 of the concept of system (comparative exchange): Regardless of size and category, the system development can be described by the exchanging level made by both the system and the external world through the index of intensity, frequency, scope and depth in terms of the information, material, energy, ideas, etc.). [7] [Note 4]

Inference 3 of the concept of system (closed exchange): The main exchange chain of the system could be closed. [7] [Note 5]

Six basic exchange elements: Material, energy, information, time, space, and spirit. [8]

The establishment of the exchange chain concept and the pull in of the spiritual elements would make the system exchange theory logically sound, make the old system theory to be more active, change it from the static situation to the dynamic process which taking the six elements including material, energy, information, time, space and spirit. In other words, the dynamic process philosophy thinking would bring us a magic system exchange theory and a new point of view that we are living in the ocean of the exchange system with full of chains and exchange elements everywhere and even with our hearts directly involved into the exchange process. What a picture of the world will this be? This will be a new one quite different from that in our minds before! This would be a new picture of the world, in which our thinking participates and gets feed back, the future life of human beings could be described, another kind ontological picture would be 
presented. For the time being, we define this picture as the chain field thinking space.

\section{Chain Field Thinking Space}

Fig. 1 shows an ideal chain field thinking space model based on one points and six directions. In this thinking space, each system is marked as a point, between any two points there are 6 elements. The actual chain field thinking space is more complicated than this model. First, rather than the 6 exchange, a system in fact would have $\mathrm{N}$ times of exchange. Secondly, the exchange activity among the systems usually occur in various kinds of way, some between the interfacing one, some striding over with others, some going with a large area. So, it is too much complicated to show it by a kind of model, what we would usually do is to think about that in our heads or to explain by high level mathematics. [9]
In this highly abstract thinking space, there are some new explain about the features of the system, such as the completeness, randomness, uncertainty. we would have a clear abstract expressing way to explain the happen, development and the evolution of the system. With this abstract thinking space model, we can even find out a mathematical method to quantitatively describe the system and its evolution.

With the specific application of the system exchange principle, we can well explain some people and things happened in the history, supplying another theory support for policy formulation, making us able to maintain a good vision of the overall situation. The system exchange theory would bring us another channel to connect the philosophy and the science, seeing the science with the height of philosophy, explaining the philosophy with scientific precision.

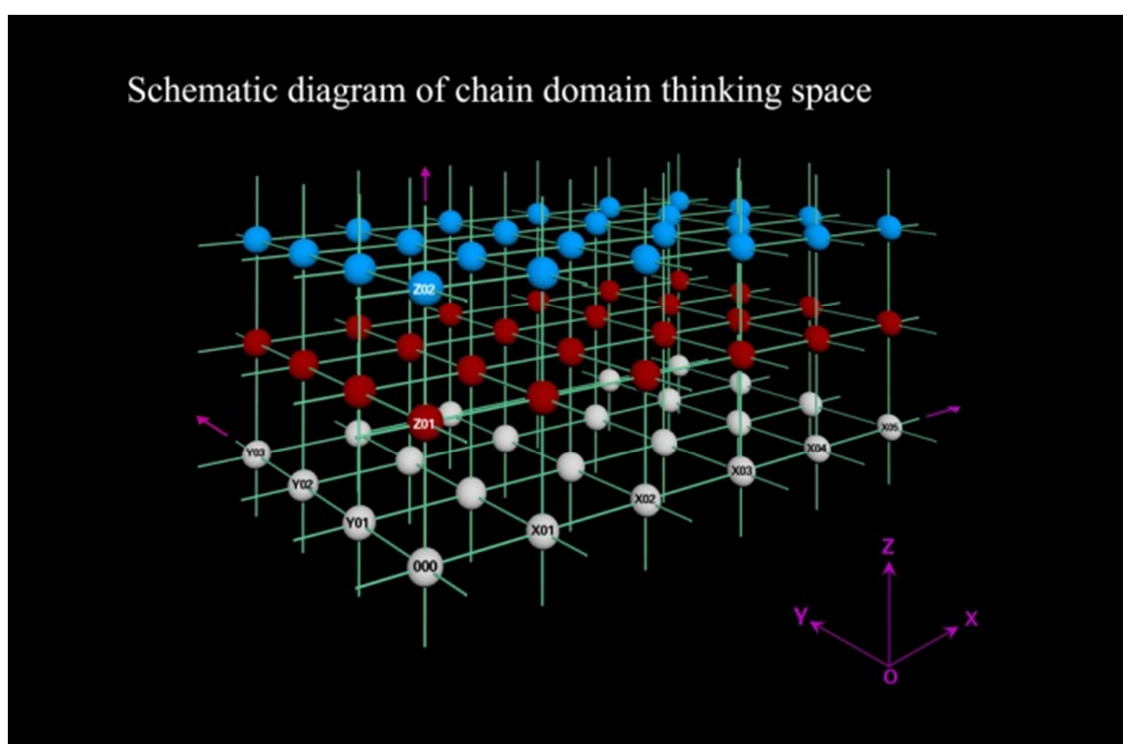

Figure 1. 3D ideal chain domain thinking space.

\section{Seven-Layer Model}

As mentioned above, the pull in of the spiritual elements actually would provide the systema thinking characteristic at the time when the system is being observed. The human brain is an independent system with the independent thinking characteristic. However, the formation of the human brain results from the long-term evolution of the nature with hundreds stage by stage and layer by layer. Therefore, we can imagine that the human brain thinking was definitely not formed in the last stage of evolution. It must be formed during the transition and evolution process from the previous inorganic layer to the organic layer and finally to the life layer. Gradually, this evolution process would produce conditioned reflexes, induction, consciousness, spirit and thinking. The evolution may still be ongoing in nowadays. Therefore, a certain sub-system or a certain element of the system would produce a kind of consciousness, this consciousness would infiltrates into a certain part of the system exchange, finally, the system would have a thinking characteristic because of the feature of the consciousness. It should be emphasized that the system we are talking about here refers not only to the human brain, but also to the living biological system in the lower organisms, even the inorganic, organic matter, and the most general system form. As long as a certain part of this exchange system infiltrates into the consciousness, this system has a systematic thinking characteristic.

However, some people may ask how the spirit or consciousness is born, how to judge the production of the consciousness in the system exchange?

In the chain field thinking space, we could conduct consciousness experiments. In the experiment, we can always find out the seven different layered distributions of the system from the inorganic world to the generation and formation of the pure spiritual forms. They can:

(1) Perform conditioned reflex or not;

(2) Record and conditioned reflex;

(3) Record, perform conditioned reflex and induce into a certain consciousness

(4) Record, induce into a certain consciousness and 
perform conditioned reflex as per this consciousness;

(5) Record, perform conditioned reflex, induce into a certain consciousness and perform conditioned reflex based on this consciousness;

(6) Organize and induce a group of consciousness, theorize it (spirit), and subconsciously apply this theorized result (spirit) to make the system have a kind of generation of the consciousness. This consciousness has the elements like material, energy, information, time, space, spirit, etc., this is a response to the feeling or a group of feelings in the full exchange process ;

(7) Consciously apply the existing theoretical results (spirit) to make the plan and take action.

We call these seven different distribution layers as the seven-layer model. From the third layer, where the system begins forming a certain consciousness, to the seventh layer where finally a pure spirit formed, We can see that the system could actually think in the different layer and by different way. In other words, the thinking we often talk about is not only performed in our brains, but in more systems, these systems usually have the function of thinking, which presented as the normal existence status.

This seven-layer model reveals the entire evolution process from a system consisting of pure material elements to a system consisting of pure spiritual elements. The whole process begins with a system of pure material elements, and ends with a system of pure spiritual elements. In fact, the various system concepts that we discuss could have their place in the seven-layer model, for instance, the solar system, earthquakes, volcanoes etc belongs to the pure material movement systems; major part of the low level living things has small part of the consciousness; the human society would combine the high level consciousness with the material; the system project and the artificial intelligence would combine the material and the consciousness; there is also the pure spiritual movement system in our brains.

\section{Existence-Thinking Dual Property of the System}

The seven-layer model would reveal the existence-thinking dual property of the exchange system, meaning that the system has existence and thinking features. This is another sublimation of people's understanding of the system, also a logical necessity for people to add the spirit element into the theory of the system. We could say that it is really not easy for people to get to know this point of view. Now, many scholars still take the system regarded as the material subconsciously at least, although they verbally express that the system is not the same thing as the material, but in their deep minds or with their thinking habits, they would still see the system same as the material. For example, the so-called complex system concept is essentially a system in which spiritual elements are involved. As even the most complicated system, it still has its rules, people can recognize it as a simple system after recognizing the rules, So, there is no complex system really existed. About 2000 years ago, people ever thought the universe very complicated, even dominated by the God. But the modern understanding of the universe is undoubtedly not the same one as before, we shall not say that there has been a kind of complicated system to stop people's cognition development.

From the above discussion, we have realized that the exchange is the existence of the system. The discussion of the seven-layer model makes us realized that when the system evolves to a certain level, it will form a systematic thinking. It should be emphasized that the evolution of the system at a certain level not necessarily refers to the emergence of the human brain, instead, it means that as long as there are enough conditioned reflections and some induction, some kind of consciousness would come out from the system. With the feature of the consciousness, this system may be far below the human brain level, but the consciousness has been produced in the system. We can certainly say that this system with the feature of the consciousness is something like the thinking. Therefore, Du Weiming ${ }^{(3)}$ said: "The energy of life (which is called qi in Chinese philosophy) that exists at all levels of the evolutionary process is both spiritual and material." [10]

With the concept of system thinking, people can classify the systems in an orderly manner. The fundamental reason that people have different opinions on the definition of the system for a long time is because that the people's understanding of the system is still at a very shallow level, with less sufficient understanding of the essence of the system. For example, regarding the interrelationships among the four major knowledge of the system theory including system engineering, system science, system philosophy, and system theory, there has been no such definition that can well unify all those four kinds. Especially for the system engineering, some people even already took it out of the system theory category. In real life, the scholars engaged in the research on system engineering and in the system philosophy are almost not related. The system study Mr. Qian Xuesen ${ }^{(4)}$ ever strongly advocated in China during his lifetime was almost aborted, and the system theory is far from being established.

With the concept of existence-thinking duality, people should have a new understanding about the relationship between material and spirit. We can no longer simply classify which the fist is between the material and spirit, or simply put the label of "sole" on the materialism or the idealism. The relationship between the material and spirit, on different levels, is of relying on each other. With only the material movement and no spiritual intervention, we cannot feel the material movement, or understand the evolution of the material system. Conversely, with only spiritual exercise and no material, we can observe and record nothing, with nothing accumulated just like there is no movement. The reason that the people could get improvement in seeing the world systematicly, in finding out the unity in the contradiction, in experiencing the world as a combined one with the material and spirit together rather than a purely material one, is just because that the material and spirit are complementary and cannot be separated. People should know that every step of progress in the cognition comes from the systematic thinking. [10].

The existence is systematic, while the thinking is a kind of system. Every step of the system evolution is resulted from the interaction between the existence and the thinking. Every exchange of the 
system toward to the external world can be regarded as a flow of elements, a present of the system existence. But the reason and mode of that flow are the result of the thinking. Recording, selection, comparison and decision-making are the so-called spiritual movements.

\section{Conclusions}

From the philosophy concepts of "interaction" and "unity of the things in the world", we could recognize the similarity and difference between the concept of system and the concept of material, through observation, induction and thought experiments. The world is actually broken. We shall point out that the essence of the system is the exchange, which is the core concept of the system theory, based on this, a new definition for the system would come out, the system exchange theory could be established. Furthermore, the chain field thinking space and the seven-layer model could be abstracted from the exchange system theory, this is also a logical necessity after the introduction of the spiritual elements into the system. From the discussion of the chain field thinking space and the seven-layer model, we can found out the existence-thinking duality of the system, so, we can dissolve the philosophy dispute between the materialism and the idealism, finally, the existence-thinking duality of the system would bring people's thinking into an ontological way.

The $\mathrm{N}$ times of the circulation moved by the logical exchange in the system would produce the whole logical exchange chain in people's thinking, by which, people would get a kind of new taste of the ontology in the philosophy and a clear prospect of human's development through the study of the history related to the people's society and the world, both of which are multi-level, multi-fields, and multi-form.

\section{Notes}

[Note 1] In many of the system definitions that have been given, I think that the "composite of several elements mutually interacting" given by Bertalanfy is the closest to the essence of the system. The system definition I present changes "mutual interaction" to "mutual exchange", which means "composite of several elements mutually exchanging". [11]

[Note 2] Although the definition of "composite of several elements mutually exchanging" is concise and clear, it is ambiguous. Therefore, using this definition with the concept of an exchange chain in actual applications may make the definition clearer. The exchange elements in the definition should be understood as the philosophical abstraction of the six elements, including material, energy, information, time, space and spirit.

[Note 3] This inference for concept of system directly interprets the system as the original driving force for the system to become bigger and stronger, and does not discuss why the system has such a driving force.

[Note 4] This inference for concept of system compares and describes the system with four virtual physical quantities, including intensity, frequency, breadth and depth. Of course, not only these four virtual physical quantities can be used to describe the system. Other quantities, such as dimensions, are also very suitable for describing the concept of system.

[Note 5] The exchange is divergent or closed, which is relative to time and space. In principle, the system exchanges, forms an exchange chain, and ultimately becomes closed.

\section{References}

[1] [US] John L. Casti. The Real World - How Computer Simulation Changes the Boundaries of Science [M]. Translated by Wang Qianxiang, Quan Lining. Shanghai: Shanghai Science and Technology Education Press, 1998: Preface.

[2] Wu Jie. System Philosophy [M]. Beijing: People's Publishing House, 2008: 43.

[3] Min Jiayin. The Pluralism of Evolution [M]. Beijing: China Social Sciences Press, 2012: 581.

[4] Wu Tong. The Broken Concept of System [J]. Journal of Systems Science, 2010(1):9.

[5] [US] Stephen Hawking, Leonard Mlodinow. Big Design [M]. Translated by $\mathrm{Wu}$ Zhongchao. Hunan: Hunan Science and Technology Press, 2011: 34.

[6] Xu Bizhen, Li Huaijun. New Theory of Universal Connection [M]. Henan: Henan University Press, 1991: 2.

[7] Yuan Hongjian. Introduction to System Exchange [J]. Journal of Systems Science, 2010(2): 88-91.

[8] Yuan Hongjian. On the Elements of Exchange Chain in System Paradigm [J]. Journal of Wuhan University of Technology (Social Science Edition), 2013(4): 638.

[9] Yuan Hongjian. Systematicism: The Integrity, Randomness and Uncertainty of Chain Domain Thinking Space [J]. Journal of Systems Science, 2017(3): 20-21.

[10] Du Weiming. Spiritual Humanism - Self, Group, Earth, Heaven [A]. Proceedings of the 24th World Philosophy Conference (Chinese version) [C]. Bern: International Federation of Philosophical Organizations, 2018: 372,372.

[11] [US] Von Bertalanfy. General System Theory - Foundation, Development, Application [M]. Lin Kangyi, Wei Hongsen, et al. Beijing: Tsinghua University Press, 1987: 51.

\section{Biography}

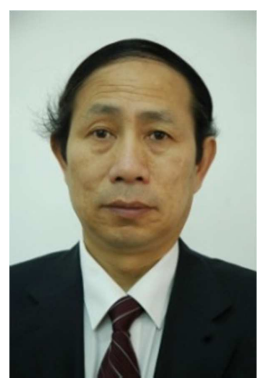

Yuan Hongjian, male, born in March 1954, Wuhan China, member of China System Science Research Association, member of China Photographers Association, member of Chinese Photographers Society, currently a visiting professor at Wuhan University of Science and Technology, research direction: system science exchange field. 